\title{
Invisible neutrino decays at the MOMENT experiment
}

\author{
Jian Tang ${ }^{* 1}$, Tse-Chun Wang ${ }^{1}$, Yibing Zhang ${ }^{1,2}$ \\ ${ }^{1}$ School of Physics, Sun Yat-Sen University, Guangzhou 510275, China \\ ${ }^{2}$ School of Mathematical and Physical Sciences, University of Sussex, United of Kingdom \\ E-mail: tangjian5email.sysu.edu.cn
}

\begin{abstract}
We investigate invisible decays of the third neutrino mass eigenstate in future accelerator neutrino experiments using muon-decay beams such as MuOn-decay MEdium baseline NeuTrino beam experiment (MOMENT). MOMENT has outstanding potential to measure the deficit or excess in the spectra caused by neutrino decays, especially in muon neutrino/antineutrino disappearance channels. Such an experiment will improve the constraints of the neutrino lifetime. Compared with exclusion limits in the current accelerator neutrino experiments T2K and NOvA under the stable neutrino assumption, we expect that MOMENT gives the better bound. The non-decay scenario is expected to be excluded by MOMENT at a confidence level of more than $3 \sigma$, if the best fit results in T2K and NOvA are confirmed. We further find that reducing systematic uncertainties is more important than the running time. Finally, we find some impact of neutrino invisible decays on the precision measurement of other oscillation parameters.
\end{abstract}

The 21st international workshop on neutrinos from accelerators (NuFact2019)

August 26 - August 31, 2019

Daegu, Korea

${ }^{*}$ Speaker. 


\section{Introduction}

The framework of three-generation neutrino oscillation has been well established through solar, atmospheric, accelerator and reactor neutrino experiments [1, 2, 3, 4]. In this paradigm, neutrino oscillations are dominated by two mass-squared splittings (i.e., $\Delta m_{31}^{2}, \Delta m_{21}^{2}$ ) and three mixing angles (i.e. $\left.\theta_{12}, \theta_{13}, \theta_{23}\right)$ [5]. Up to now, most of the oscillation parameters have been measured well [6], except the Dirac CP phase $\delta$ and the neutrino mass ordering (normal mass hierarchy: $\Delta m_{31}^{2}>0$; inverted mass hierarchy: $\Delta m_{31}^{2}<0$ ). The current precision measurement in the quark sector reaches the sub-percent level and serves as a tool to probe new physics. Following the same strategy, it is natural to expect near future neutrino oscillation experiments such as JUNO [7], T2HK [8], DUNE [9] and MOMENT [10] to search for new physics beyond threegeneration neutrino oscillations including sterile neutrinos $[11,12,13]$, non-standard neutrino interactions $[14,15,16,17]$ and neutrino decays $[18,19,20,21]$.

Neutrino decays are classified into invisible and visible scenarios. In the visible decay scenario, decay products can be detected by the detector, like $v_{j} \rightarrow \bar{v}_{i}\left(v_{i}\right)+J$ have been put forward, where $J$ denotes a Majoron. If the final states of neutrino decays are unobservable to the detector, those decays are called invisible decays [21]. Given that neutrinos are Dirac particles, the coupling gives rise to neutrino decay products: $v_{i} \rightarrow \bar{v}_{j R}+\chi$, where $\chi$ is a light iso-singlet scalar and $v_{i R}$ is a right-handed fermion [19, 23]. Majorana neutrinos lead to decay products [24, 25], $v_{j} \rightarrow v_{4}+J$. The $v_{2}$ decay in the invisible channel has been constrained well from solar neutrino oscillation data, which gives the bound $\tau_{2} / m_{2}>7.2 \times 10^{-4} \mathrm{~s} / \mathrm{eV}$ at $90 \%$ C.L. [26]. Recently a combined analysis of NOvA and T2K data pointed to a result of $\tau_{3} / m_{3}>1.5 \times 10^{-12} \mathrm{~s} / \mathrm{eV}$ along with the constraints by an individual experiment: $\tau_{3} / m_{3} \geq 7 \times 10^{-13} \mathrm{~s} / \mathrm{eV}$ in NOvA and $\tau_{3} / m_{3} \geq 1.41 \times 10^{-12} \mathrm{~s} / \mathrm{eV}$ in T2K [27].

Compared with the superbeam experiment by pion decays, MOMENT will offer clean neutrino sources from muon decays to probe new physics with a baseline detector using Gd-doped water cherenkov technology, which have been demonstrated to have excellent properties to study new physics. In this work [28], we focus on invisible neutrino decays and demonstrate how the eigenstate $v_{3}$ decay would affect precision measurements of standard neutrino mixing parameters.

\section{Simulation of neutrino invisible decays at MOMENT}

We assume that the neutrino decay products are sterile neutrinos and the mass ordering is given by $m_{3}>m_{2}>m_{1}>m_{4}$ as the following decay process: $v_{3} \rightarrow v_{4}+J$. The connection between flavour eigenstates and mass eigenstates can be given as:

$$
\left(\begin{array}{l}
v_{\alpha} \\
v_{s}
\end{array}\right)=\left(\begin{array}{ll}
U & 0 \\
0 & 1
\end{array}\right)\left(\begin{array}{l}
v_{i} \\
v_{4}
\end{array}\right)
$$

The Hamiltonian of neutrino propagation in matter can be written as:

$$
H=U\left\{\frac{1}{2 E}\left(\begin{array}{ccc}
0 & 0 & 0 \\
0 & \Delta m_{21}^{2} & 0 \\
0 & 0 & \Delta m_{31}^{2}
\end{array}\right)-i \frac{m_{3}}{2 E \tau_{3}}\left(\begin{array}{lll}
0 & 0 & 0 \\
0 & 0 & 0 \\
0 & 0 & 1
\end{array}\right)\right\} U^{\dagger}+\left(\begin{array}{ccc}
2 \sqrt{2} G_{F} N_{e} E & 0 & 0 \\
0 & 0 & 0 \\
0 & 0 & 0
\end{array}\right),
$$



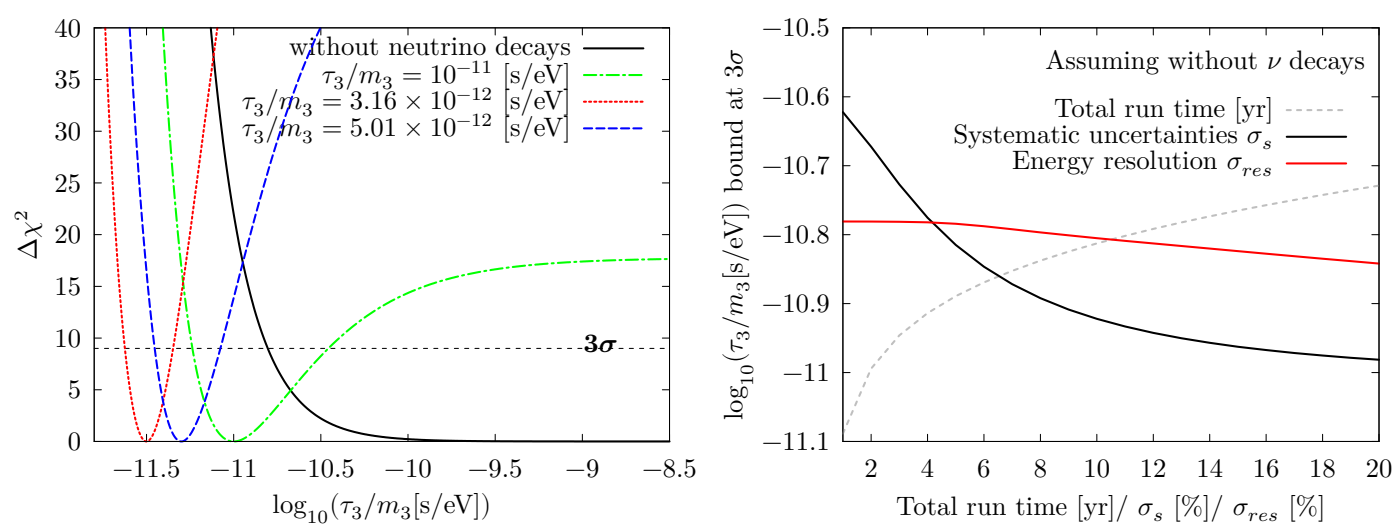

Figure 1: The left panel shows $\Delta \chi^{2}=\chi^{2}-\chi_{\min }^{2}$ as a function of the test value of $\tau_{3} / m_{3}$. The input (true) value of $\tau_{3} / m_{3}$ is assumed to be stable (black solid), $10^{-11}$ (green dashed-dotted), $5.01 \times 10^{-12}$ (blue shortdashed) and $3.16 \times 10^{-12} \mathrm{~s} / \mathrm{eV}$ (red dotted) at MOMENT. The right panel presents constraints at $3 \sigma$ on $\tau_{3} / m_{3}$ for the stable-neutrino case against the total running time (the short-dashed grey curve), the size of systematic uncertainty $\sigma_{s}$ (black), and the energy resolution $\sigma_{r e s}$ (red). We run experiments from 1 to 20 years, while $\sigma_{s}$ and $\sigma_{r e s}$ vary in the range $[1 \%, 20 \%]$ [28].

where $U$ is the PMNS mixing matrix [29, 30], $G_{F}$ is the Fermi coupling constant, $N_{e}$ is the electron density, $E$ is the neutrino energy and $\tau_{3}$ is the lifetime of $v_{3}$. Obviously, probabilities in neutrino and antineutrino modes remain invariant after a replacement of $\delta \rightarrow-\delta$ and $N_{e} \rightarrow-N_{e}$, i.e. $P_{v_{\alpha} \rightarrow v_{\beta}}\left(E, L ; \delta, N_{e}\right)=P_{\bar{v}_{\alpha} \rightarrow \bar{v}_{\beta}}\left(E, L ;-\delta,-N_{e}\right)$. Numerical oscillation probabilities were done by diagonalizing the Hamiltonian matrix [31]. The simulation details for MOMENT were given in Ref. [17, 13] with eight oscillation channels: $v_{e} \rightarrow v_{e}, v_{e} \rightarrow v_{\mu}, v_{\mu} \rightarrow v_{e}, v_{\mu} \rightarrow v_{\mu}$ and their $\mathrm{CP}$-conjugate partners. The major backgrounds are mostly from the atmospheric neutrinos, neutral current backgrounds and charge mis-identifications. They can be largely suppressed by the beam direction and proper modelling of background spectra within the beam-off period. Our simulation was carried out with the help of GLoBES $[32,33]$. The central values and their uncertainties for the standard oscillation parameters were taken from the NuFit4.0 [6].

When we switch on neutrino decays with $\tau_{3} / m_{3}=10^{-12} \mathrm{~s} / \mathrm{eV}$, a clear difference between the cases with and without invisible neutrino decays could be easily observed in simulations. Invisible decays washed out the extreme of neutrino oscillations, where the differences in $v_{\mu}$ and $\bar{v}_{\mu}$ disappearance channels were more significant than the other channels. This could affect the precision measurement of neutrino mixing parameters such as $\theta_{23}$ and $\Delta m_{31}^{2}$ which are mostly involved in these channels. In other words, the other channels could help with a clarification of this bias induced by neutrino decays.

In Fig. 1, we show the constraint on $\tau_{3} / m_{3}$ for four different true values. As the true value of $\tau_{3} / m_{3}$ gets smaller, the constraint becomes tighter. It is obvious that the larger neutrino decays take place, the tighter constraint we can obtain. The appearance of the upper bound at $3 \sigma$ is significant, which did not show up in the current measurements by T2K and NOvA. We note that the behaviour of $\Delta \chi^{2}$ looks symmetric for $\tau_{3} / m_{3}=3.16 \times 10^{-12}$ [s/eV], but does not for the larger value of $\tau_{3} / m_{3}$. It is because in that case, $\Delta \chi^{2}$ is approaching to $\sim 120$ when neutrino is stable. The range of $\Delta \chi^{2}$ shown in Fig. 1 is near the bottom. We find an important result by comparing 

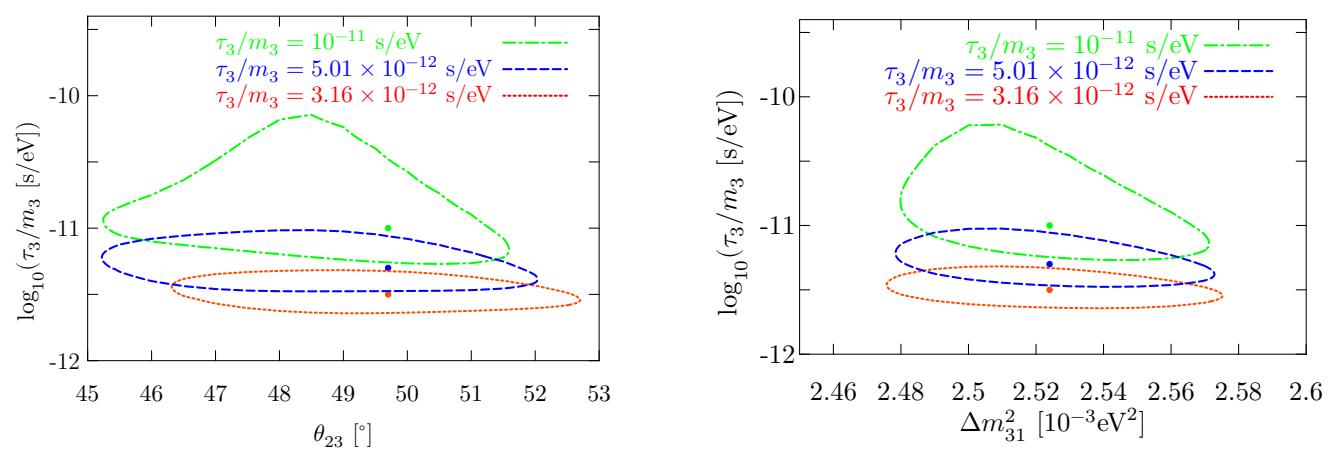

Figure 2: The exclusion contour at $3 \sigma$ on the planes any two of $\log _{10}\left(\tau_{3} / m_{3}[\mathrm{~s} / \mathrm{eV}]\right)$ and $\theta_{23}$ (left) and $\Delta m_{31}^{2}$ (right). We show for different true values: $\tau_{3} / m_{3}=10^{-11}$ (dashed-dotted green), $5.01 \times 10^{-12}$ (short-dashed blue) and $3.16 \times 10^{-12}$ (dotted red) s/eV [28].

the impact of the total running time and energy resolution $\sigma_{s}$. We can see improving $\sigma_{s}=1 \%$ can improve better $\left.\left(\log _{10}\left(\tau_{3} / m_{3}\right)[\mathrm{s} / \mathrm{eV}]\right)=-10.6\right)$ than that by doubling the total running time $\left.\left(\log _{10}\left(\tau_{3} / m_{3}\right)[\mathrm{s} / \mathrm{eV}]\right)=-10.7\right)$.

Thanks to the precision measurement of $\tau_{3} / m_{3}$, we can obtain a closed contour in Fig. 2 instead of a wide band as what we saw in the combined fitting result of T2K and NOvA in Ref. [27]. On average, the precision at $3 \sigma$ in $\theta_{23}$ is almost $3-3.5^{\circ}$ at MOMENT. We also observe some impact from the true value of $\tau_{3} / m_{3}$ on the $\theta_{23}$ measurement. The uncertainty of $\Delta m_{31}^{2}$ at $3 \sigma$ C.L. is about $0.05 \times 10^{-3} \mathrm{eV}^{2}$. The impact on $\theta_{23}$ is more pronounced than the mass squared difference here.

\section{Summary}

Neutrino decays lead to suppression and damping effects in the framework of three-generation neutrino oscillations, and could be measured in the precisely reconstructed spectra at the MOMENT experiment. The $v_{\mu}$ and $\bar{v}_{\mu}$ disappearance channels are more important than other channels while focusing on the maximum or minimum is a good strategy to scrutinize these effects caused by neutrino decays. We have simulated the MOMENT experiment and found outstanding potential to constrain the $\tau_{3} / m_{3}$ parameter. Given the best-fit values hinted by T2K and NOvA [27], we have found that MOMENT would improve the precision measurement of invisible neutrino decays. If the current best fit discovered in [27] is confirmed, the standard non-decay scenario can be excluded at a confidence level of more than $3 \sigma$. The projections of $\theta_{23}-\log _{10}\left(\tau_{3} / m_{3}\right)$ and $\Delta m_{31}^{2}-$ $\log _{10}\left(\tau_{3} / m_{3}\right)$ have demonstrated little correlations between $\theta_{23}$ and $\Delta m_{31}^{2}$ at $3 \sigma$ confidence level.

We have further investigated the impact of statistical and systematic uncertainties by varying the total running time, changing the size of the normalisation uncertainty $\sigma_{s}$ and energy resolution respectively. By increasing the total running time or reducing the systematic uncertainties, we will improve the sensitivity in invisible neutrino decays. Reducing systematic uncertainties is more important than increasing the total running time in the MOMENT experiment. Neither is it important to improve energy resolution in the detector. 


\section{References}

[1] B. Aharmim et al. [SNO Collaboration], Phys. Rev. C 88, 025501 (2013).

[2] R. Wendell et al. [Super-Kamiokande Collaboration], Phys. Rev. D 81, 092004 (2010).

[3] S. Abe et al. [KamLAND Collaboration], Phys. Rev. Lett. 100, 221803 (2008).

[4] F. P. An et al. [Daya Bay Collaboration], Phys. Rev. D 95, no. 7, 072006 (2017).

[5] C. Patrignani et al. [Particle Data Group], Chin. Phys. C 40, no. 10, 100001 (2016).

[6] I. Esteban, M. C. Gonzalez-Garcia, M. Maltoni, I. Martinez-Soler and T. Schwetz, JHEP 1701, 087 (2017).

[7] F. An et al. [JUNO Collaboration], J. Phys. G 43, no. 3, 030401 (2016).

[8] K. Abe et al. [Hyper-Kamiokande Collaboration], arXiv:1805.04163 [physics.ins-det].

[9] R. Acciarri et al. [DUNE Collaboration], arXiv:1512.06148 [physics.ins-det].

[10] J. Cao et al., Phys. Rev. ST Accel. Beams 17, 090101 (2014).

[11] P. Minkowski, Phys. Lett. 67B, 421 (1977).

[12] K. N. Abazajian et al., arXiv:1204.5379 [hep-ph].

[13] J. Tang, Y. Zhang and Y. F. Li, Phys. Lett. B 774, 217 (2017)

[14] M. B. Gavela, D. Hernandez, T. Ota and W. Winter, Phys. Rev. D 79, 013007 (2009).

[15] F. Bonnet, D. Hernandez, T. Ota and W. Winter, JHEP 0910, 076 (2009).

[16] M. B. Krauss, T. Ota, W. Porod and W. Winter, Phys. Rev. D 84, 115023 (2011).

[17] J. Tang and Y. Zhang, Phys. Rev. D 97, no. 3, 035018 (2018).

[18] C. W. Kim and W. P. Lam, Mod. Phys. Lett. A 5, 297 (1990).

[19] A. Acker, S. Pakvasa and J. T. Pantaleone, Phys. Rev. D 45, 1 (1992).

[20] A. Acker, A. Joshipura and S. Pakvasa, Phys. Lett. B 285, 371 (1992).

[21] M. Lindner, T. Ohlsson and W. Winter, Nucl. Phys. B 607, 326 (2001).

[22] S. Pakvasa, AIP Conf. Proc. 542, no. 1, 99 (2000).

[23] A. Acker and S. Pakvasa, Phys. Lett. B 320, 320 (1994).

[24] G. B. Gelmini and M. Roncadelli, Phys. Lett. 99B, 411 (1981).

[25] Y. Chikashige, R. N. Mohapatra and R. D. Peccei, Phys. Lett. 98B, 265 (1981).

[26] R. Picoreti, M. M. Guzzo, P. C. de Holanda and O. L. G. Peres, Phys. Lett. B 761, 70 (2016).

[27] S. Choubey, D. Dutta and D. Pramanik, JHEP 1808, 141 (2018).

[28] J. Tang, T. C. Wang and Y. Zhang, JHEP 1904, 004 (2019) [arXiv:1811.05623 [hep-ph]].

[29] Z. Maki, M. Nakagawa and S. Sakata, Prog. Theor. Phys. 28, 870 (1962).

[30] B. Pontecorvo, Sov. Phys. JETP 6, 429 (1957) [Zh. Eksp. Teor. Fiz. 33, 549 (1957)].

[31] T. Hahn, physics/0607103.

[32] P. Huber, M. Lindner and W. Winter, Comput. Phys. Commun. 167, 195 (2005).

[33] P. Huber, J. Kopp, M. Lindner, M. Rolinec and W. Winter, Comput. Phys. Commun. 177, 432 (2007). 